\title{
Observational signatures of hot-star magnetospheres
}

\author{
Mary E. Oksala ${ }^{1,2}$ \\ ${ }^{1}$ Department of Physics, California Lutheran University \\ email: moksala@callutheran.edu \\ ${ }^{2}$ LESIA, Observatoire de Paris
}

\begin{abstract}
Magnetic fields play an important role in shaping the circumstellar environment of hot, massive stars. Observational diagnostics give clues to the presence of magnetism across the entire electromagnetic spectrum. Infrared features can show more complex structure, indicating they may probe deeper opacities than optical features. Optical and infrared features mimic each other, with identical blue and red peak variations and identical peak velocity of material. These comparisons indicate the location of the infrared and optical emitting material is similar. Longer wavelength diagnostics are currently being developed and tested. IR spectroscopy is a viable tool to detect magnetic candidates in the Galactic center and star forming regions.
\end{abstract}

Keywords. techniques: spectroscopic, infrared: stars, stars: early-type, stars: magnetic fields

Optical and infrared spectra identify material trapped in the star's magnetosphere, detected via hydrogen recombination lines; the emission pattern depends on magnetic and stellar properties (i.e. field strength, mass-loss rate, stellar geometry).

\section{Motivation}

- Identify tools to understand dynamics and structure of massive star magnetospheres

- Explore the possibility of infrared to probe deeper through optically thick regions

- Confront differences between observation and theory. Can infrared reveal structures theoretically predicted, but undetected in optical?

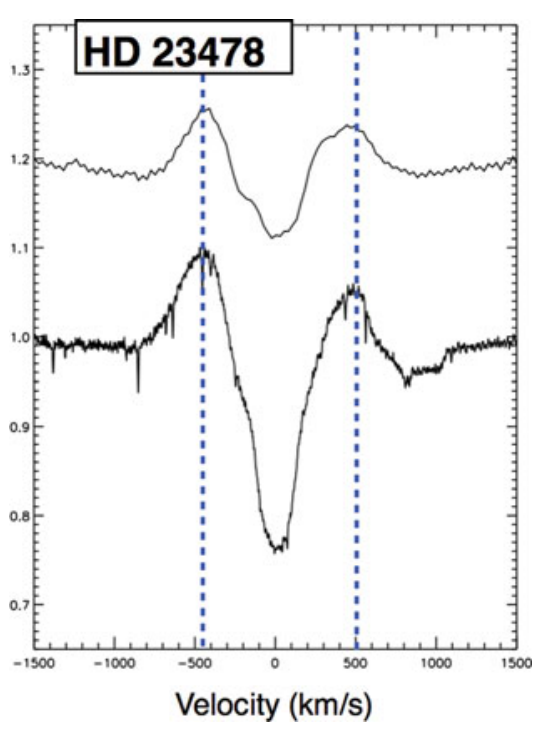

Figure 1. Spectral features for the magnetic, rapidly-rotating B-type star HD 23478. The star was identified as a magnetic candidate due to its emission features in the infrared, prior to confirmation of the presence of a magnetic field. The $\mathrm{Br} \gamma$ line (top, Gemini/GNIRS, $\mathrm{R} \sim 6000$ ) and the $\mathrm{H} \alpha$ line (bottom, TBL/Narval, $\mathrm{R} \sim 65000$ ) exhibit similar line shape and maximum peak velocity. The blue dashed lines indicate the peak velocity in the optical. These comparisons indicate the location of the infrared and optical emitting material is similar. The decreased stellar flux in the infrared could make the emission features more easy to observe, and allowing for detection of lower density magnetospheres, which remain invisible in the optical. 